\title{
Kinetics of Observable with Power-law Mixing Rule in the Second-order Reaction Process
}

\author{
Hisashi YAMAMOTO

\begin{abstract}
Department of Food Science and Nutrition, Faculty of Human Life and Science, Doshisha Women's College of Liberal Arts, Kamigyo-Ku, Kyoto 602-0893, Japan
\end{abstract}

\begin{abstract}
Some biopolymer gelation process shows an inflection point (IP) in a growth curve of a rheological observable. In this note, a kinetic treatment is presented for such a second-order food reaction process $d x / d t=K_{2}(1-x)^{2}$ where a general observable $o$ grows with reaction degree $x$ through the power-law type mixing rule $o^{\nu}=(1-x) o_{\mathrm{R}}^{\nu}+x o_{\mathrm{P}}^{\nu}$. For $-1<\nu<1$, the observable-time curve could possess IP at $o^{*}=(1-\nu)^{1 / \nu}(1+\nu)^{-1 / \nu} o_{\mathrm{P}}$ and $K_{2} t^{*}=2^{-1}(1+\nu) \nu^{-1}\left(1-\left(o_{\mathrm{R}} / o_{\mathrm{P}}\right)^{\nu}\right)-1$ with the maximum growth rate $d o /\left.d t\right|_{t^{*}}=2^{2}(1-\nu)^{(1 / \nu)-1}(1+\nu)^{-(1 / \nu)-1} \nu\left(1-\left(o_{\mathrm{R}} / o_{\mathrm{P}}\right)^{\nu}\right)^{-1} o_{\mathrm{P}} K_{2}$. These results show that $o_{\mathrm{R}}$ dependence disappears in the product $\left(1+K_{2} t^{*}\right) d o /\left.d t\right|_{t^{*}}$. The whole $o$-t curve is linearized with the transformation $\left(o_{\mathrm{P}}^{\nu}-o_{\mathrm{R}}^{\nu}\right)\left(o_{\mathrm{P}}^{\nu}-o^{\nu}\right)^{-1}=1+K_{2} t$. The case of $\nu=0$ is compensated with a logarithmic type (ln $o$ ) mixing rule.
\end{abstract}

Key words: Kinetics, Observable, Power-law, Mixing rule, Second-order reaction

\section{Introduction}

Recently, inspired from the viscosity growth behavior observed in alkali gelatinization of rice starch [1,2], the present author provided a theoretical analysis [3] for the kinetics of an observable $o$ in the first-order reaction $(\mathrm{R} \rightarrow$ P) process $d x / d t=K_{1}(1-x)$ where $o$ grows from $o_{\mathrm{R}}$ to $o_{\mathrm{P}}$ with reaction degree $x \equiv[\mathrm{P}] /([\mathrm{R}]+[\mathrm{P}])$ through the power-law type mixing rule

$$
o^{\nu}=(1-x) o_{\mathrm{R}}^{\nu}+x o_{\mathrm{P}}^{\nu}
$$

Here the exponent $\nu$ is called a mixing parameter and characterizes the shape of observable growth curves. Combining the solution $x(t)$ of the reaction rate equation with the mixing rule (1), we demonstrated for $\nu<1$ that the observable-time curve could possess an inflection point (IP) at $o^{*}=(1-\nu)^{\frac{1}{v}} o_{\mathrm{P}}$ [3]. Subsequenly, through somewhat formal discussion, i.e. without using the explicit solution $x(t)$ of the reaction rate equation, this formula for IP was shown to be extended to $o^{*}=((1-\nu) /(1-(1-$ n) $\nu))^{1 / \nu} o_{\mathrm{P}}$ for general $n$-th order reaction [4].

Among general higher-order $(n \geq 2)$ reactions, however, the actual food reaction that most likely occurs would be of second-order

$$
\frac{d x}{d t}=K_{2}(1-x)^{2}
$$

Here $K_{2}(>0)$ denotes a rate constant. Such a secondorder reaction is often seen in biopolymer gelation caused by binary collisions of polymer chains [5]. For most readers, it might therefore be more helpful to show the concrete procedure for the kinetic treatment of the second-order reaction (2), as was done for the first-order reaction [3].

For example, Lopes da Silva et al. studied the gelation process of high-methoxyl pectins/sucrose system by means of dynamic rheological measurement [6] and obtained at an isothermal condition the storage modulus $\left(G^{\prime}\right)$-time curves possessing IP. They regarded the gelation process as a second-order reaction, but did not estimate the rate constant $K_{2}$ through a kinetic procedure for the whole isothermal $G^{\prime}-t$ data. In order to study the rate constant through the analysis of $G^{\prime}-t$ data, one must anyhow relate the rheological observable with the reaction rate equation (2). This role could be played by the power-law mixing rule of observable in Eq. (1).

In this note, we would present a kinetic treatment of an increasing observable $\left(o_{\mathrm{R}}<0<o_{\mathrm{P}}\right)$ in such a second-order reaction process with the power-law mixing rule (1). Although some results for IP will be included in the previous ones $(n=2)$ derived for a general-order case [4], we expect the contents of this note to serve for an actual kinetic analysis of the second-order food reaction system.

(Received 17 Dec. 2006: accepted 19 Jan. 2007) 


\section{The second-order kinetics of observable with power-law mixing rule}

In our argument [3,4], it is convenient to introduce 'relative observable' $O \equiv o / o_{\mathrm{P}}$ and 'relative proper observable' $\tilde{O} \equiv o_{\mathrm{R}} / o_{\mathrm{P}}<1$. In terms of these dimensionless parameters the mixing rule (1) reads as

$$
O^{\nu}=\tilde{O}^{\nu}+\gamma x
$$

where a constant $\gamma$ is defined as $\gamma \equiv 1-\tilde{O}^{\nu}$ and satisfies the relation $\nu^{-1} \gamma>0$ for any $\nu \neq 0$.

With the initial condition $x(0)=0$, the rate equation (2) has the solution $x(t)=K_{2} t /\left(1+K_{2} t\right)$. Inserting it into Eq (3), we get

$$
O(t)^{\nu}=\tilde{O}^{\nu}+\gamma K_{2} t\left(1+K_{2} t\right)^{-1}
$$

from which follows the growth rate of relative observable

$$
\frac{d O}{d t}=\nu^{-1} \gamma K_{2} O^{1-\nu}\left(1+K_{2} t\right)^{-2}
$$

The second derivative of $O$ is then calculated with the result

$$
\frac{d^{2} O}{d t^{2}}=\nu^{-1} \gamma K_{2}^{2}\left(1+K_{2} t\right)^{-3} O^{1-\nu} D(O)
$$

with $D(O) \equiv\left(1-\nu^{-1}\right)\left(1-O^{-\nu}\right)-2$, where we have utilized the relation $\gamma\left(1+K_{2} t\right)^{-1}=1-O^{\nu}$ derived from Eq. (4) and the definition of $\gamma$.

In the right-hand side of Eq. (6), a product of factors except $D(O)$ is positive definite and so the sign of the second derivative is the same with that of $D(O)$. The factor $D(O(t))$ depends monotonically on time, and takes at $t=\infty$ $D(O(\infty)=1)=-2<0$ implying that the $O-t$ curve is always convex near the equilibrium. Therefore, if and only if the curve is concave at $t=0$, a unique IP can exist at some finite time $t=t^{*}$. This concavity condition $D(O(0)=$ $\tilde{O})>0$ is expressed with the following inequality

$$
1+\nu^{-1}<\left(\nu^{-1}-1\right) \tilde{O}^{-\nu}
$$

When a mixing parameter $\nu$ is positive, the inequality (7) is transformed to $1+\nu<(1-\nu) \tilde{O}^{-\nu}$, for which one finds no solution in the region $\nu \geq 1$. In the region $0<\nu<$ 1 , the concavity condition holds if and only if relative proper observable $\tilde{O}$ is small enough to satisfy the inequality

$$
o_{\mathrm{P}}>\left(\frac{1+\nu}{1-\nu}\right)^{\frac{1}{\nu}} o_{\mathrm{R}}
$$

Next, when a mixing parameter $\nu$ is negative valued, the condition (7) reads as $1+\nu>(1-\nu) \tilde{O}^{-\nu}$, for which one finds no solution in the region $\nu \leq-1$. In the region $-1<$ $\nu<0$, the condition is reduced to the same form as Eq. (8).
Thus, for $-1<\nu<1$, the observable-time curve indicates under the condition (8) a concave behavior in the early stage $\left(0 \leq t \leq t^{*}\right)$ of reaction process. At IP $\left(t=t^{*}\right)$, $D\left(O=O^{*}\right)$ should be zero. This fact leads to $O^{*}=((1-$ $\nu) /(1+\nu))^{1 / \nu}$, i.e.

$$
o^{*}=\left(\frac{1-\nu}{1+\nu}\right)^{\frac{1}{\nu}} o_{\mathrm{P}}
$$

Eqs. (8) and (9) are nothing but the special $(n=2)$ cases of those previously derived for general $n$-th order reaction [4]. Eq. (9) indicates that the relative observable $O^{*}(\nu)$ at IP in the second-order reaction is invariant under the transoformation $\nu \leftrightarrow-\nu$ and is bounded with the upper limit $O^{*}(\nu \rightarrow 0)=e^{-2}=0.1353 \cdots$ (Fig. 1$)$.

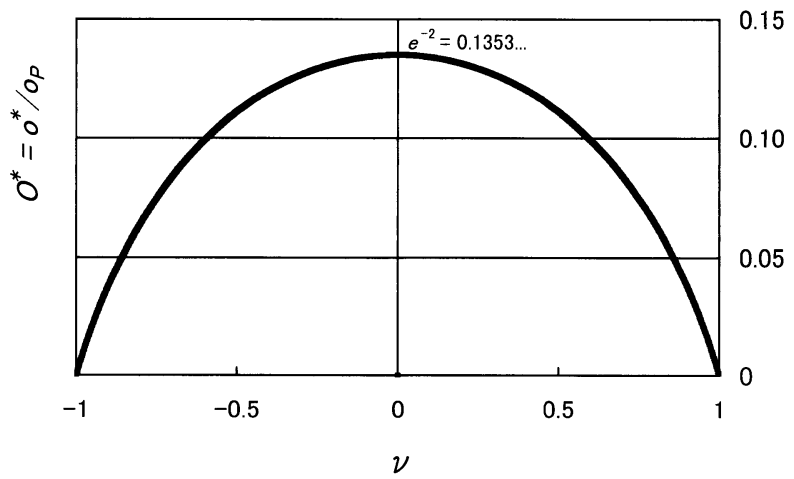

Fig. 1 Dependence of the relative observable $O^{*}=o^{*} / o_{\mathrm{P}}$ at IP on the mixing parameter $\nu . O^{*}$ takes the maximum $\left(e^{-2}=\right.$ $0.1353 \cdots)$ at $\nu=0$.

The inverse function of $O^{*}(\nu)$ is therefore two valued. Comparing the value of $((1-\nu) /(1+\nu))^{1 / \nu}$ with that of $\left(o^{*} / o_{\mathrm{P}}\right)\left(<e^{-2}\right)$ observed experimentally, one could then determine the absolute value $|\nu|$ for the mixing parameter. Two values $\pm|\nu|$ shall be selected as the candidates for $\nu$.

One should note here that for this estimation of $|\nu|$ we do not need to know $o_{\mathrm{R}}$. In the case that the measurement of $o$ is carried out in the full process $(\mathrm{R} \rightarrow \mathrm{P})$ of reaction, $o_{\mathrm{P}}$, the value at the equilibrium would generally be easier to detect than $o_{\mathrm{R}}$ which must be observed at the initial time of reaction.

From Eq. (9) and the mixing rule (3) follows the reaction degree at IP: $x^{*}=1-2(1+\nu)^{-1} \nu \gamma^{-1}$. Then, the time at IP is obtained from the solution $x(t)$ of Eq. (2) as

$$
K_{2} t^{*}=2^{-1}(1+\nu) \nu^{-1} \gamma-1
$$

By use of these results and Eq. (5), the first-derivative of $O$ at IP, i.e. the maximum growth rate of relative observable is derived with the result

$$
\left.\frac{d O}{d t}\right|_{t^{*}}=2^{2}(1-\nu)^{\frac{1}{\nu}-1}(1+\nu)^{-\frac{1}{\nu}-1} \nu \gamma^{-1} K_{2}
$$


As shown in Eqs. (10) and (11), both expressions of $t^{*}$ and $d O /\left.d t\right|_{t^{*}}$ include $\gamma$, which means that both depend on $o_{\mathrm{R}}$ as well as on the rate constant $K_{2}$. If $o_{\mathrm{R}}$ can be known experimentally, then for each value of $\nu= \pm|\nu|$ predicted from $o^{*} / o_{\mathrm{P}}$ with Eq. (9), the value of $K_{2}$ can be conjectured, for example, from the time at IP by solving Eq. (10).

If some technical diffculty would arise in the measurement of $o_{\mathrm{R}}$, one should note the following product

$$
\left.\left(1+K_{2} t^{*}\right) \frac{d O}{d t}\right|_{t^{*}}=2(1-\nu)^{\frac{1}{\nu}-1}(1+\nu)^{-\frac{1}{\nu}} K_{2},
$$

where disappears the dependence on $o_{\mathrm{R}} . K_{2}$ can then be computed from $t^{*}$ and $d O /\left.d t\right|_{t^{*}}$ by solving Eq. (12), and it is also possible to infer the value of $o_{\mathrm{R}}$ from Eq. (10).

In this way, we would have two sets of kinetic parameters $\left(\nu, K_{2}\right)$ (and of $\left.o_{\mathrm{R}}\right)$ predicted only from the data $\left(o^{*} / o_{\mathrm{P}}\right.$, $\left.t^{*}, d O /\left.d t\right|_{t^{*}}\right)$ observed experimentally at IP and at the equilibrium.

In Fig. 2 are shown the examples of relative observabletime curves with typical choices $(\nu=1,0.5,0,-0.5,-1)$ of

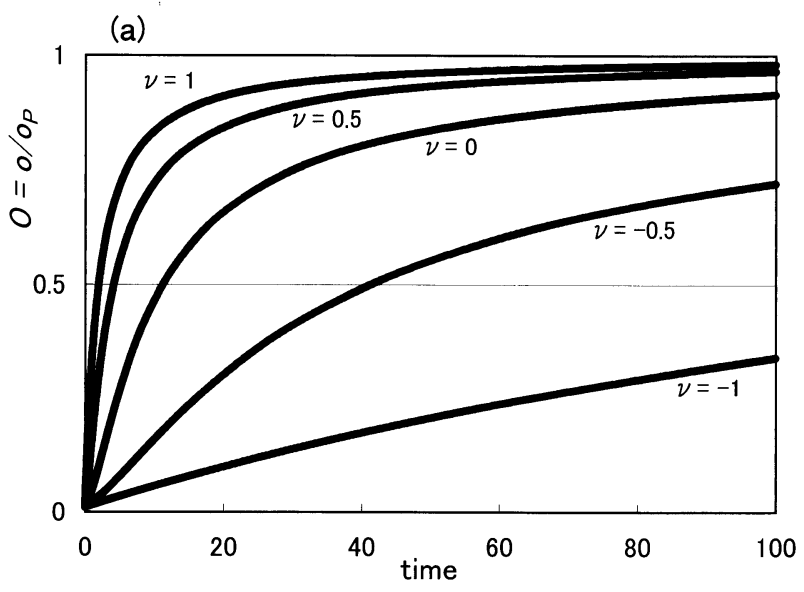

(b)

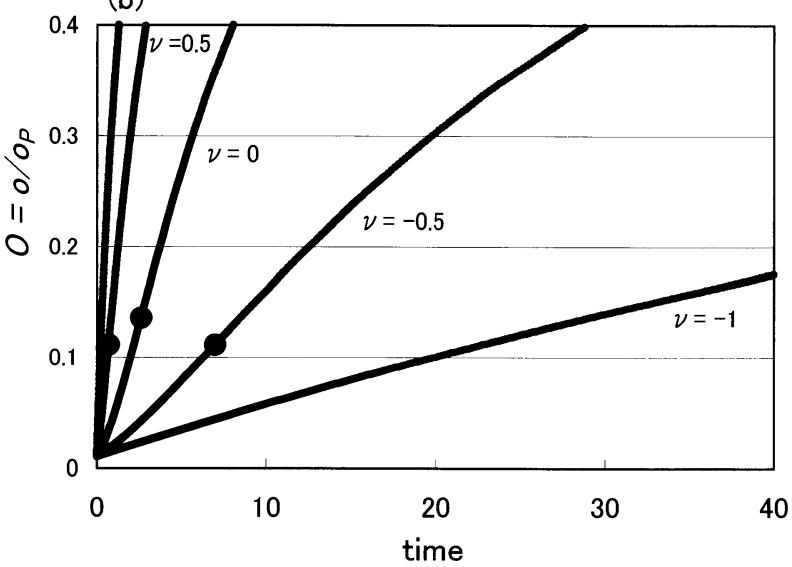

Fig. 2 The examples of relative observable-time curves with typical choices $(\nu=1,0.5,0,-0.5,-1)$ of a mixing parameter (a). A rate constant $K_{2}$ and relative proper observable $\tilde{O}=o_{\mathrm{R}} / o_{\mathrm{P}}$ are set to be 0.5 and 0.01 respectively. The curves are enlarged in (b) with solid circles indicating IP for $\nu=0.5,0$ and -0.5 . a mixing parameter. Here the case with $\nu=0$ is exceptional and shall be treated separately in the next section.

\section{A logarithmic case}

An exceptional case $(\nu=0)$ of the power-law rule (1) is compensated with the logarithmic rule [3,4]

$$
\ln o=(1-x) \ln o_{\mathrm{R}}+x \ln o_{\mathrm{P}}
$$

In terms of the dimensionless variables, this reads

$$
\ln O=(1-x) \ln \tilde{O}
$$

Putting the second-order solution $x(t)$ into Eq. (14) and taking the first and the second derivatives with respect to time, we obtain

$$
\begin{gathered}
\frac{d O}{d t}=\left(\ln \tilde{O}^{-1}\right) K_{2}\left(1+K_{2} t\right)^{-2} O \\
\frac{d^{2} O}{d t^{2}}=\left(\ln \tilde{O}^{-1}\right) K_{2}^{2}\left(1+K_{2} t\right)^{-3} O\left(\ln O^{-1}-2\right)
\end{gathered}
$$

Eqs. (15) and (16) indicate that under the condition $\ln \tilde{O}^{-1}$ $>2$, i.e. $o_{\mathrm{P}}>e^{2} o_{\mathrm{R}}$, the observable-time curve possesses IP at which $o^{*}=e^{-2} o_{\mathrm{P}}, x^{*}=1-2\left(\ln \tilde{O}^{-1}\right)^{-1}, t^{*}=K_{2}^{-1}\left(2^{-1} \ln \tilde{O}^{-1}\right.$ $-1)$ and $d O /\left.d t\right|_{t^{*}}=2^{2} e^{-2}\left(\ln \tilde{O}^{-1}\right)^{-1} K_{2}$. One can check that all the results for the power-law rule obtained in Sec. 2 . coincide in the $\nu \rightarrow 0$ limit with those for the above logarithmic rule, which provides the maximum of $O^{*}(\nu)$. If the experimentally measured value of $o^{*} / o_{\mathrm{P}}$ would coincide with $e^{-2}=0.1353 \cdots$, the mixing rule will uniquely be chosen to be the logarithmic one. Then, it is possible to compute the rate constant from the relation $\left(1+K_{2} t^{*}\right) d O /\left.d t\right|_{t^{*}}=$ $2 e^{-2} K_{2}$ and also the value of $o_{\mathrm{R}}$ from $t^{*}$.

\section{Linearization of the whole curve}

As seen above, except for the logarithmic case, the kinetic parameters $\left(\nu, K_{2}\right)$ cannot uniquely be determined solely from the data at IP. Also, it would in any case be desirable to incorporate the whole data points into the analysis to predict confidently the rate constant of reaction process. For this purpose, it is useful to linearize the whole observable-time curve.

For $\nu \neq 0$, we can easily derive from Eq. (4) the following relation

$$
\left(o_{\mathrm{P}}^{\nu}-o_{\mathrm{R}}^{\nu}\right)\left(o_{\mathrm{P}}^{\nu}-o^{\nu}\right)^{-1}=1+K_{2} t
$$

For the whole observable-time data, plotting the quantity on the left-hand side of Eq. (17) computed with each value of $\nu$, i.e. $\pm|\nu|$ already chosen from the data at IP and the equilibrium, one could estimate again $K_{2}$ with a 
linear regression analysis. If the measurement of $o_{\mathrm{R}}$ would suffer from some difficulty, one could use the value calculated from Eq. (10).

Then, from the theoretical consistency, namely, from the linearity of $\left(o_{\mathrm{P}}^{\nu}-o_{\mathrm{R}}^{\nu}\right)\left(o_{\mathrm{P}}^{\nu}-o^{\nu}\right)^{-1}-t$ data and the coincidence of $K_{2}$ between those predicted from IP and from Eq. (17), one will be able to judge which value $( \pm|\nu|)$ should be a suitable one as the mixing paremeter. The reliable value of $K_{2}$ should finally be determined from the analysis of the whole curve.

Similary, the whole curve characterized with the logarithmic rule (14) can be linearized with the relation

$$
\left(\ln o_{\mathrm{P}}-\ln o_{\mathrm{R}}\right)\left(\ln o_{\mathrm{P}}-\ln o\right)^{-1}=1+K_{2} t
$$

that will serve for estimating $K_{2}$ from the whole $o-t$ data.

In the actual application to the second-order reaction such as gelation of biopolymers, a general observable $o$ might be replaced with some rheological variable such as a storage (loss) modulus $G^{\prime}\left(G^{\prime \prime}\right)$. However, the analysis procedure presented above could in principle apply to any second-order reaction system with any growing observable including non-rheological ones.

\section{References}

[1] H. Yamamoto, E. Makita, Y. Oki, M. Otani; Flow characteristics and gelatinization kinetics of rice starch under strong alkali conditions. Food Hydrocolloids, 20, 9-20 (2006).

[2] H. Yamamoto, N. Isozumi, T. Sugitani; A concavity property of the viscosity growth curve during alkali gelatinization of rice starch. Carbohydrate Polym., 62, 379-386 (2005).

[3] H. Yamamoto; Kinetics of observable with power-law mixing rule in the first-order reaction process. Jpn. J. Food Eng., 7, 245-248 (2006).

[4] H. Yamamoto; An inflection point in the observable growth curve for general-order reaction process with power-law mixing rule. In submission to publication in Jpn. J. Food Eng..

[5] S. B. Ross-Murphy; The estimation of junction zone size from geltime measurements. Carbohydrate Polym., 14, 281-294 (1991).

[6] J. A. Lopes da Silva, M. P. Goncalves, M. A. Rao; Kinetics and thermal behaviour of the structure formation process in HMP/sucrose gelation. Int. J. Biol. Macromol., 17, 25-32 (1995) 